\title{
Ushering in a new era: human papillomavirus (HPV) testing comes to the NHS Cervical Screening Programme
}

\author{
Anne Szarewski
}

Centre for Cancer Prevention, Wolfson Institute for Preventive Medicine, Queen Mary, University of London, London, UK

\section{Correspondence to} Dr Anne Szarewksi, Centre for Cancer Prevention, Wolfson Institute for Preventive Medicine, Queen Mary, University of London, Charterhouse Square, London EC1M 6BQ, UK; anne.szarewski@qmul.ac.uk

Received 10 February 2011 Accepted 17 February 2011

\section{Background}

The National Health Service Cervical Screening Programme (NHSCSP) has announced that from April 2011, human papillomavirus (HPV) testing will be incorporated into the national screening programme in England. Initially, HPV testing will be used for the triage of borderline and mildly dyskaryotic smears, but there are also plans, at a later date, for its use as a 'test of cure' following treatment for cervical abnormalities (Figure 1). ${ }^{1}$ It would have been interesting to see the data underpinning the proposals, but no references were given.

The announcement follows 8 years of pilot projects. The first pilots were completed in $2006^{2}$ and were followed by the Sentinel Site Implementation Project, which was launched in January 2008, to examine the practical implementation of a national roll-out of HPV testing. A report from the latter does not appear to be publicly available, so it is not possible to tell whether the problems identified in the pilots have been overcome and, if so, how. It is thus impossible to judge the results of the changes.

\section{HPV triage}

The majority of low-grade cytological abnormalities (at least 70\%) will regress without treatment. ${ }^{3}$ If it was possible to identify those women whose abnormalities were very likely to regress, they could be spared the anxiety of repeat testing and colposcopy referral. Testing for high-risk HPV types is currently the most effective way of doing this: a negative HPV test means there is very little chance of any abnormality being present. ${ }^{3}$ The introduction of liquid-based cytology into the NHSCSP paved the way for 'reflex' testing of cytology samples, in which those showing borderline or mild dyskaryosis could automatically have an HPV test performed on their sample, without the need for the woman to be re-examined. If highrisk HPV is found they will be referred for colposcopy; if HPV is not found they will be returned to routine screening.

Why has the NHSCSP chosen to use triage in the management of women with mild, as well as borderline, dyskaryosis? The American College of Obstetrics and Gynecology published guidelines on the use of HPV triage in 2009, but only recommended its use in women with atypical squamous cells of unknown significance (ASCUS) (roughly equivalent to borderline) smears, since a very high proportion of women with low-grade squamous intraepithelial lesions (LSIL) (equivalent to mild dyskaryosis) would test positive and be referred for colposcopy anyway. ${ }^{4}$ This is borne out by the results of the pilots, in which HPV positivity rates were $46 \%$ for women with borderline smears and $83 \%$ for those with mild dyskaryosis. ${ }^{2}$ Similar figures were shown in the UK ARTISTIC study, in which HPV positivity rates were $31 \%$ for women with borderline smears and $86 \%$ for those with mild dyskaryosis. ${ }^{5}$ HPV positivity is higher in young women, reflecting the high incidence of transient infections, and indeed, in the pilots, two centres decided not to continue to refer HPV-positive women under the age of 35 years directly for colposcopy, because the number of referrals had more than doubled. In the ARTISTIC trial, HPV positivity rates in women aged $25-29$ years were $48 \%$ in women with borderline dyskaryotic smears. ${ }^{5}$

It would therefore appear that the majority of women with a single borderline or mildly abnormal smear will in fact be referred straight to colposcopy. How will the clinics cope if they suddenly have a large increase in the number of referrals? 


\section{HPV Triage and \\ Test of Cure Protocol \\ For women aged 25 to 64 years}

Cancer Screening Programmes

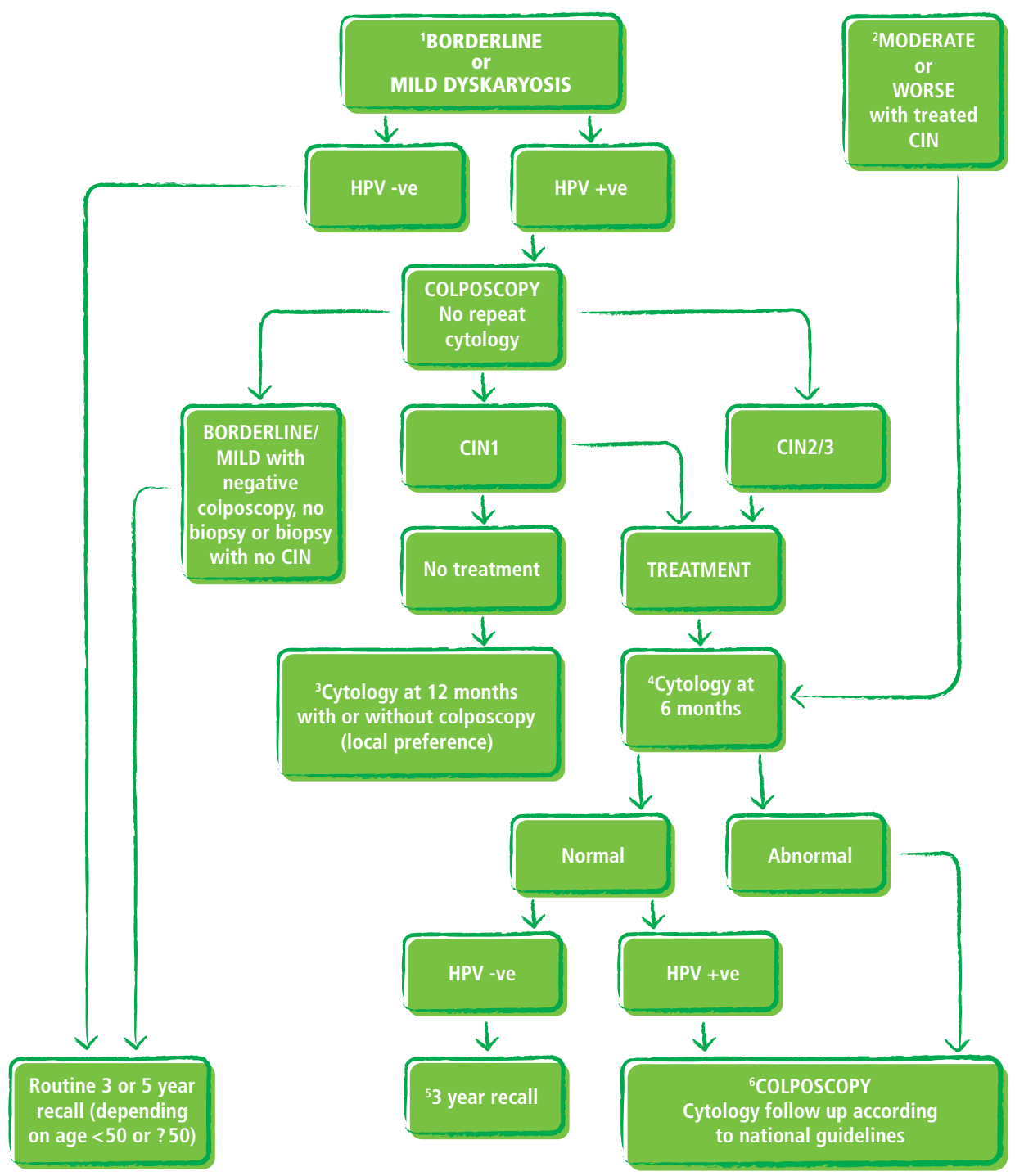

1 If sample is unreliable/inadequate for the HPV test, refer mild and recall borderline for 6 month repeat cytology. At repeat cytology HPV test if Neg/Bord/Mild. If HPV negative return to routine recall, refer if HPV positive. Refer moderate or worse cytology.

2 Untreated CIN1 should be managed as per untreated CIN1 following borderline/mild.

3 Follow up of 12 month cytology only should follow normal NHSCSP protocols.

4 Women in annual follow up after treatment for CIN are eligible for the HPV test of cure at their next screening test.

5 Women $\geq 50$ who have normal cytology at 3 years will then return to 5 yearly routine recall.

6 Women referred due to borderline/mild cytology or normal cytology/HPV positive, who then have a satisfactory and negative colposcopy can be recalled in three years.

N.B Women who reach 65 still require to complete the protocol and otherwise comply with national guidance.

HPV Triage and Test of Cure Protocol April 2010

Figure 1 National Health Service Cervical Screening Programme (NHSCSP) flow diagram of 'HPV Triage and Test of Cure Protocol for Women Aged 25 to 64 Years'. ${ }^{1}$ Figure available at: http://www.cancerscreening.nhs.uk/cervical/hpv-triage-test-flowchart-v2.pdf. ${ }^{1}$ This flow diagram is reproduced with the kind permission of the NHSCSP. 
Admittedly, colposcopy clinics have become less busy since the decision to raise the screening age to 25 years, but many will also have fewer staff as a result. It is likely that there will be an increase in the detection of highgrade cervical intraepithelial neoplasia (CIN), both due to the higher sensitivity of HPV testing compared to cytology and to the fact that fewer women are lost to follow-up. This is to be welcomed. However, it should not be forgotten that, in the pilots, women who were HPV-positive were significantly more anxious, distressed and concerned about their test results, mainly because they did not understand what their results meant. ${ }^{2}$ It could be argued that, since then, the introduction of HPV vaccination should have improved public knowledge around HPV; unfortunately, while awareness is certainly higher, knowledge does not appear to be much greater. ${ }^{6}$ More education of both the public and health professionals is still needed.?

The triage programme is to start in April 2011, but in reality only the current Sentinel Sites (Sheffield, Manchester,Liverpool,Bristol, Norwich and Northwick Park Hospital in London) are actually ready. There are many issues to be resolved elsewhere; laboratories will need to be reorganised and equipment purchased, staff will need training and administrative practices will need to be altered. In practice, then, in the rest of the country, the national programme is unlikely to even start being rolled out before October 2011.

\section{Test of cure}

In addition to HPV testing in triage, an HPV 'test of cure' is being introduced (though it is not clear exactly when) for women who have undergone treatment for CIN. The proposal is that women will have cytology performed 6 months after their treatment; if the result is negative, an HPV test will be carried out, using the same cytology sample. If the HPV test is negative, women will be discharged back to routine recall in 3 or 5 years; if they are aged over 60 years, this would mean they do not attend again (since recall ceases at aged 64 years). If, however, the HPV test is positive or cytology screening does show an abnormality then the women will be referred back to colposcopy (Figure 1). ${ }^{1}$

Many clinicians have been eagerly awaiting the introduction of HPV 'test of cure' as there is evidence that HPV negativity is a good predictor of disease clearance. However, studies suggest that it often takes longer than 6 months to clear HPV infection after treatment, which means women will be worried unnecessarily by positive tests. ${ }^{8} 9$ A recent Australian study found that while over 90\% of HPV 16 and 18 infections cleared within approximately 24 months post-treatment, only around $50 \%$ had cleared within 6 months of treatment. ${ }^{10}$ A UK study that followed women for 2 years suggested that a strategy of HPV testing at 6 months might be safe, but this was carried out in three centres of excellence, with an extremely low residual disease/recurrence rate. ${ }^{11}$ Several studies of residual disease rates after large loop excision of the transformation zone (LLETZ) treatment have demonstrated that negative excision margins are associated with lower risk and positive excision margins with higher risk of residual disease but this does not appear to be taken into account in the new management algorithm (Figure 1). ${ }^{1}$ Meanwhile, most of the HPV 'test of cure' studies have only followed up women for around 2 years. Can we be sure it is safe to return these women to routine screening when it is known that they continue to be at higher risk of developing cancer for at least 10 years? ${ }^{12} 13$

Funding The author has received honoraria for lecturing, consulting fees and conference sponsorship from GlaxoSmithKline, Sanofi-Pasteur, Merck, Sharp and Dohme, Bayer Schering and Wyeth.

Competing interests The author is an investigator in the Cervarix trials. She is also Editorin-Chief of the Journal of Family Planning and Reproductive Health Care.

Provenance and peer review Not commissioned; externally peer reviewed.

\section{References}

1 National Health Service Cervical Screening Programme (NHSCSP). HPV Triage and Test of Cure Protocol for Women Aged 25 to 64 Years. April 2010. http://www.cancerscreening. nhs.uk/cervical/hpv-triage-test-flowchart-v2.pdf [accessed 8 February 2011].

2 Moss SM, Gray A, Marteau T, et al. Evaluation of HPV/LBC. Cervical Screening Pilot Studies. First Report to the Department of Health on Evaluation LBC (December 2002). http://www. cancerscreening.nhs.uk/cervical/lbc-pilot-evaluation.pdf [accessed 8 February 2011].

3 Wright TC Jr, Massad LS, Dunton CJ, et al.; 2006 American Society for Colposcopy and Cervical Pathology-sponsored Consensus Conference. 2006 consensus guidelines for the management of women with cervical intraepithelial neoplasia or adenocarcinoma in situ. Am J Obstet Gynecol 2007;197:340-345.

4 American College of Obstetricians and Gynecologists (ACOG). ACOG Practice Bulletin: Cervical cytology screening. Obstet Gynecol 2009;114:6.

5 Kitchener H, Almonte M, Gilham C, et al. ARTISTIC: a randomised trial of human papillomavirus (HPV) testing in primary cervical screening. NIHR Health Technol Assess J 2009;13:51. http://www.hta.ac.uk/project/1162.asp [accessed 8 February 2011].

6 Williams K, Forster A, Marlow L, et al. Attitudes towards human papillomavirus vaccination: a qualitative study of vaccinated and unvaccinated girls aged $17-18$ years. J Fam Plann Reprod Health Care 2011;37:22-25.

7 Szarewski A. Social and psychological aspects of cervical screening. Expert Rev Obstet Gynecol 2011;6:37-44.

8 Chan BK, Melnikow J, Slee CA, et al. Posttreatment human papillomavirus testing for recurrent cervical intraepithelial neoplasia: a systematic review. Am J Obstet Gynecol 2009;200:422.e1-422.e9.

9 Kreimer AR, Katki HA, Schiffman M, et al.; ASCUS-LSIL Triage Study Group. Viral determinants of human papillomavirus 
persistence following loop electrical excision procedure treatment for cervical intraepithelial neoplasia grade 2 or 3 . Cancer Epidemiol Biomarkers Prev 2007;16:11-16.

10 Moore EE, Danielewski JA, Garland SM, et al. Clearance of human papillomavirus in women treated for cervical dysplasia. Obstet Gynecol 2011;117:101-108.

11 Kitchener HC, Walker PG, Nelson L, et al. HPV testing as an adjunct to cytology in the follow up of women treated for cervical intraepithelial neoplasia. BJOG 2008;115:1001-1007.
12 Soutter WP, Sasieni P, Panoskaltsis T. Long-term risk of invasive cervical cancer after treatment of squamous cervical intraepithelial neoplasia. Int J Cancer 2006;118:2048-2055.

13 National Health Service Cervical Screening Programme (NHSCSP). Colposcopy and Programme Management: Guidelines for the NHS Cervical Screening Programme (2nd edn) (NHSCSP Publication No. 20). May 2010. http://www. cancerscreening.nhs.uk/cervical/publications/nhscsp20.html [accessed 8 February 2011]. 